\title{
Diagonal and off-diagonal disorder in doped quantum wells
}

\author{
E. A. de Andrada e Silva, I. C. da Cunha Lima, A. Ferreira da Silva, and C. E. Leal \\ Laboratório Associado de Sensores e Materiais (LAS), Instituto de Pesquisas Espaciais (INPE), Caixa Postal 515, \\ 12201 Sao José dos Campos, São Paulo, Brazil
}

(Received 4 May 1987; revised manuscript received 20 October 1987)

\begin{abstract}
We consider the formation of an impurity band in the electronic density of states (DOS) of a quantum well of $\mathrm{Ga}_{1-x} \mathrm{Al}_{x} \mathrm{As} / \mathrm{GaAs}$. We consider both the bandwidth coming out of a fluctuation in the binding energy (diagonal disorder) and a fluctuation in the transfer matrix $\left(V_{i j}\right)$ due to randomness on the impurity locations in the plane parallel to the interfaces. We calculate the DOS for an impurity layer thickness half the well length, and show that in this case the off-diagonal disorder is dominant. A comparison with experimental results is made.
\end{abstract}

\section{INTRODUCTION}

Donor and acceptor impurity states can be made to occur in $\mathrm{Ga}_{1-x} \mathrm{Al}_{x} \mathrm{As} / \mathrm{GaAs}$ heterostructure as, for instance, the one shown in Fig. 1. For one donor impurity, either inside the small-band-gap region (GaAs) or the large-band-gap region $\left(\mathrm{Ga}_{1-x} \mathrm{Al}_{x} \mathrm{As}\right)$, an additional electron state appears below the first subband. If the impurity concentration is high enough, extended states can be occupied and a two-dimensional electron gas (2D EG) is formed inside the quantum well $(\mathrm{QW}) .^{1-7}$ In that case impurities play the role of scattering centers for the $2 \mathrm{D}$ EG. Very-high-mobility samples can be produced by modulation doping the $\mathrm{Ga}_{1-x} \mathrm{Al}_{x}$ As layer; ${ }^{8}$ in that case the 2D EG is separated from the scatterers by an undoped layer, the spacer.

Bound states associated with impurities inside the $Q W$ are important in determining the infrared properties of the heterostructure. These states have been investigated experimentally by Shanabrook and Comas. ${ }^{6}$ They have measured the binding energy of the electron and observed that this energy decreases as the well gets wider and as distance from the impurit to the well's center increases. The single impurity in the $\mathrm{QW}$ was solved theoretically by Bastard, ${ }^{1}$ where he worked out the ground-state energy variationally in the approximation of the infinite well. Since then many improvements of his work came out as reviewed by Greene and Bajaj. ${ }^{2}$ More recently multiplequantum-well structures ${ }^{9-13}$ (MQW's) have attracted much attention and there are already extensive works on it.

An interesting result from experiments is that the measured binding energy for an electron in the ground state is smaller than what has been calculated. Of course many corrections could be added, and have been added, to Bastard's calculation, like electron-phonon coupling, finite well depth, etc. Two other reasons come out of disorder arguments, as follows. It has been shown by two of us (E.A.A.S. and I.C.C.L.) that an asymmetric impurity band resulting from structural disorder in both a metaloxide-semiconductor (MOS) structure $^{14}$ and in a $\mathrm{QW}$ (Ref. 3) accounts for the decrease in the binding energy. On the other hand, as pointed out by Bastard and dis- cussed in more detail in the next section, a distribution of impurities along the well (distribution on the $z$ coordinate) gives rise also to an impurity band. This one shows an infinitely sharp peak in the lower edge. Oliveira and Falicov ${ }^{5}$ used the arguments of observable "center of gravity transitions" to explain the measured binding energy.

In this paper we are interested in studying the interplay of the two kinds of disorder: the one due to a distribution on the $z$ coordinate of the impurities, a diagonal disorder, resulting in a fluctuation of the single-site binding energy, and that one resulting from a fluctuation in the transfer matrix of the Hamiltonian due to randomness in the impurities relative positions - the off-diagonal disorder. In the next section we establish the theory for the averaged DOS including both kinds of disorder. In Sec. III we discuss and show the resulting DOS. We also investigate the effect of a nonuniform doping profile and conclude that, if the impurity layer is concentric with the well, this effect is not relevant.

\section{THEORY OF DOPED QW's}

Since the problem of an electron bound to an impurity in a QW was first treated by Bastard, many improvements on his model came out, but resulted always in

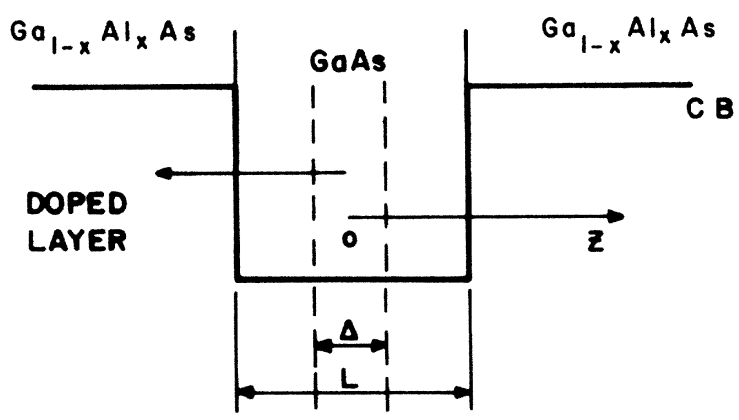

FIG. 1. Scheme of the $\mathrm{GaAs} / \mathrm{Ga}_{1-x} \mathrm{Al}_{x} \mathrm{As} \mathrm{QW}$. The well width $L$, the impurity layer thickness $\Delta$, and the $z$ direction are assigned with the origin at the center of the well. 
small corrections $(<10 \%)^{2}$ Basically he describes the electron in the well by the effective-mass approximation, attracted by an impurity at $\mathbf{r}_{i}$. The binding energy of the ground state depends on the impurity coordinate $z_{i}$ due to the confinement potential, so a dispersion on $z$ results in a dispersion on energy giving rise to a finite width in the DOS. Unless the concentration is high enough, overlapping between different impurity sites can be neglected and the model Hamiltonian for this case can be written as

$$
H=\sum_{i} \varepsilon\left(z_{i}\right) a_{i}^{\dagger} a_{i} .
$$

The DOS per impurity is independent of concentration and is given by

$$
D(\omega)=-\frac{1}{\pi} \sum_{i} \operatorname{Im} G_{i i}\left(\omega+i 0^{+}\right),
$$

where $G_{i i}$ is the single-particle propagator. If $P\left(z_{i}\right)$ describes the impurity profile, the averaged DOS becomes

$$
\bar{D}(\omega)=\int P\left(z_{i}\right) \delta\left(\omega-\varepsilon\left(z_{i}\right)\right) d z_{i} .
$$

So far we have described the origin of the impurity band obtained by Bastard and other authors in subsequent papers. ${ }^{5,13}$ Recently two of us $^{3}$ (E.A.A.S. and I.C.C.L.) treated the problem of impurity-band formation under a different approach. The impurities were assumed as distributed in a thin layer as compared to the well width $L$, and to the averaged separation $\bar{R}$ between them. So, they could be considered as lying in a plane at $z_{i}=$ const. No dispersion in $z_{i}$ occurs, but disorder appears due to the randomness of the impurity locations on the plane. In the case where the concentration is such that overlap cannot be neglected, the following model Hamiltonian can be used:

$$
H=\sum_{i} \varepsilon a_{i}^{\dagger} a_{i}+\sum_{i \neq j} V_{i j} a_{i}^{\dagger} a_{i}
$$

This 2D structural disorder in $V$, here called offdiagonal disorder, can be treated suitably, as in Ref. 15, by the method of Matsubara and Toyozawa (MT), ${ }^{16}$ which consists in a diagrammatic expansion of the single-particle propagator, in a selection of sets of selfavoiding diagrams and in assuming a uniform distribution of impurities along the plane.

A more complicated situation occurs when the impurities occupy several atomic layers in the $z$ direction and at the same time the concentration is high. The, both diagonal disorder in $z_{i}$ and structural in $V_{i j}$, must be considered. In that case the model Hamiltonian becomes

$$
H=\sum_{i} \varepsilon_{i} a_{i}^{\dagger} a_{i}+\sum_{i \neq j} V_{i j} a_{i}^{\dagger} a_{j}
$$

where we have used for short $\varepsilon_{i}=\varepsilon\left(z_{i}\right)$ and $V_{i j}=V\left(\mathbf{r}_{i}-\mathbf{r}_{j}\right)$. Then, we have two sources of disorder for $V_{i j}$ : the 2D structural disorder due to the randomness occupancy in the plane $(x, y)$ and the $z_{i}$ coordinate randomness, the latter appearing through the groundstate wave functions in the definition of $V$.

Let us assume that the impurities are located in a layer of thickness $\Delta<L$ inside a $\mathrm{QW}$ according to a profile
$P\left(z_{i}\right)$. We can always define a set of very thin sublayers, at least mathematically, as shown in Figs. 2(a) and 2(b). Were the impurities contained in a single sublayer, the problem would be reduced to the 2D structural disorder in $V_{i j}$. We could then apply, as in Refs. 3,14, and 15, the MT method in the planes represented in Fig. 2(c). However, the transfer matrix in Eq. (5) mixes impurity sites belonging to different sublayers. In that case, the DOS obeys

$$
\bar{D}_{N}(\omega) \neq \sum_{\text {sublayers }} \bar{D}_{N_{\mathrm{sl}}}^{\mathrm{MT}}(\omega),
$$

where $N_{\text {sl }}$ stands for the impurity concentration corresponding to each sublayer. Nevertheless, we might assume, to be checked $a$ posteriori, that $V_{i j}$ is not very sensitive to which sublayers the $i$ and $j$ impurities belong to, i.e., in Fig. 2(c) we can assume $V_{a b} \simeq V_{a b^{\prime}}$. This would be the case if we were in a range of concentration in which the averaged separations in the $(x, y)$ plane $\bar{R}$, between two impurities was much larger than the impurity layer thickness. Then we could have

$$
\bar{D}_{N}(\omega) \simeq \int P\left(z_{i}\right) \bar{D}_{N}^{\mathrm{MT}}\left(\omega, \varepsilon\left(z_{i}\right), V_{i j}\left(z_{i}\right)\right) d z_{i},
$$

where $\bar{D}_{N}^{\mathrm{MT}}$ would be obtained by assuming that all impurities lie in the plane of $z_{i}, P\left(z_{i}\right)$ playing the role of a weight function.

It has been shown by Bastard, and later confirmed in other more sophisticated calculations, that the binding energy changes faster with $z_{i}$ midway between the center and the barriers of the $\mathrm{QW}$ than in these latter regions. And so does, a fortiori, the $V_{i j}\left(z_{i}\right)$ that enters in Eq. (7). If the whole impurity layer lies in such a slowly varying region, or if it is thin enough, we can approximate $\bar{D}_{N}(\omega)$ by

$$
\bar{D}_{N}(\omega) \simeq \int P\left(z_{i}\right) \bar{D}_{N}^{\mathrm{MT}}\left(\omega, \varepsilon\left(z_{i}\right), V_{i j}\right) d z_{i},
$$

i.e., we can assume the strict $2 \mathrm{D}$ limit in calculating $V_{i j}$, but not in calculating $\varepsilon_{i}$. However, in the DOS obtained from the Hamiltonian like the one shown in Eq. (4), the role of the single-site energy is just to define the position of the center of the band. Therefore, we have the following symmetry property:

$$
\bar{D}_{N}^{\mathrm{MT}}(\omega, \varepsilon)=\bar{D}_{N}^{\mathrm{MT}}(\omega-\varepsilon, 0) .
$$

In consequence, Eq. (8) becomes

$$
\bar{D}_{N}(\omega) \simeq \int P\left(z_{i}\right) \bar{D}_{N}^{\mathrm{MT}}\left(\omega-\varepsilon\left(z_{i}\right)\right) d z_{i} .
$$

In order to verify the accuracy of the approximations

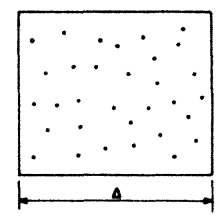

(a)

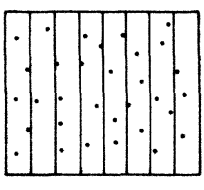

(b)

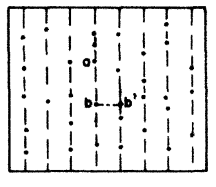

(c)
FIG. 2. Illustration of how the impurities in a layer of thickness $\Delta$ can be distributed in sublayers and of the representative planes parallel to the interfaces. In (c) the point $b$ represents the projection of the site $b^{\prime}$, in the plane of $a$. 
in Eqs. (7) and (10), we must compare the most discrepant DOS in the strict 2D limit for planes inside the impurity layers. To clarify, if the layer extends from $-\Delta / 2$ to $+\Delta / 2$, i.e., is concentric with the well, we compare the DOS in the 2D limit at $z_{i}=0$ and $z_{i}=+\Delta / 2$. Since the DOS is normalized to 1 (one state per impurity) we can define a similarity parameter by

$\eta_{a b}=\frac{1}{2} \int d \omega\left|\bar{D}_{N}^{\mathrm{MT}}\left(\omega, \varepsilon\left(z_{a}\right)\right)-\bar{D}_{N}^{\mathrm{MT}}\left(\omega, \varepsilon\left(z_{b}\right)\right)\right|$.

In the schematic plot of Fig. $3 \eta_{0, L / 2}$ corresponds to half the shaded areas and occurs in the range $0 \leq \eta \leq 1$. Then, the accuracy criteria for using Eq. (7) can be
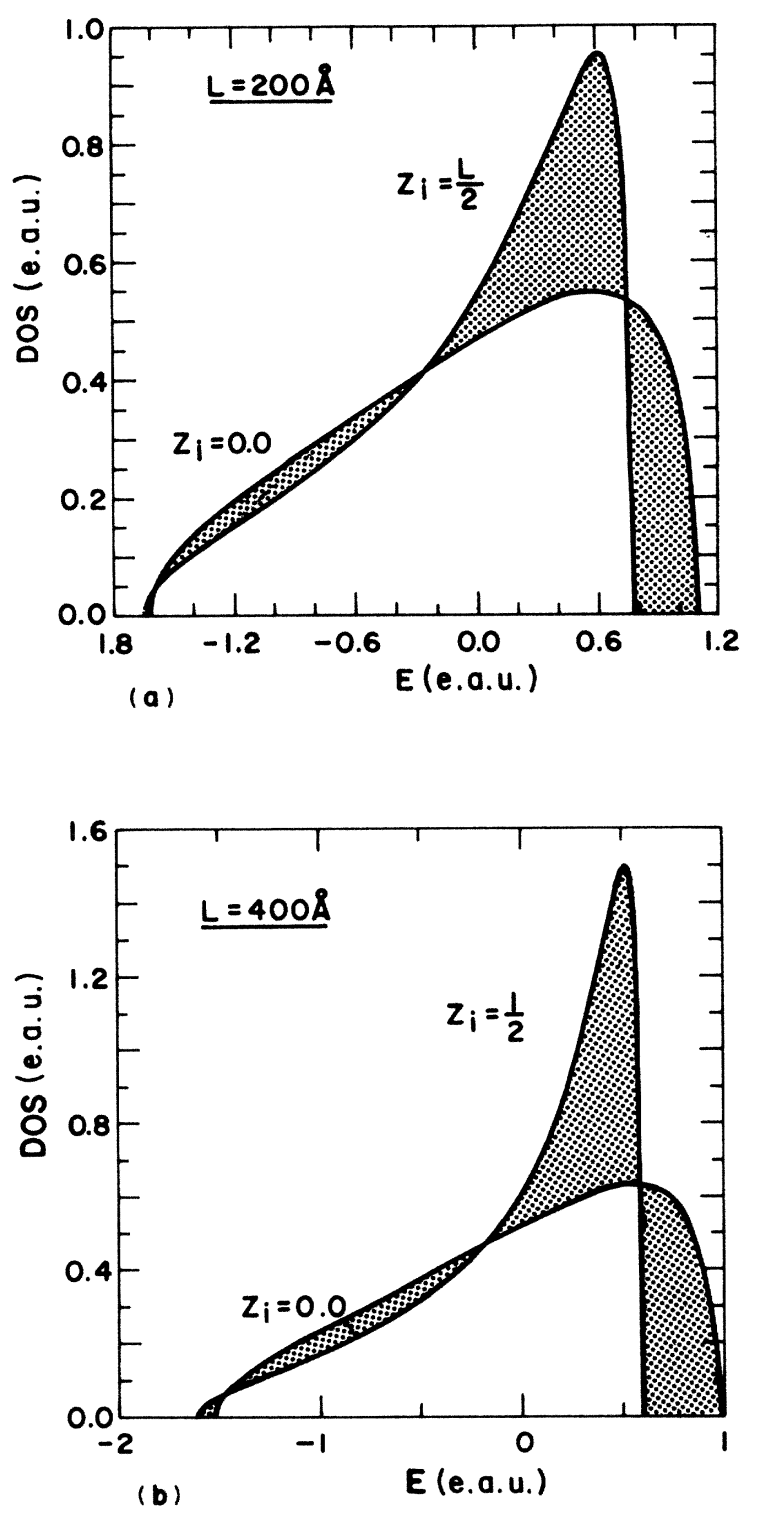

FIG. 3. Examples of the deformation of the 2D MT impurity band in $\mathrm{GaAs} / \mathrm{Ga}_{1-x} \mathrm{Al}_{x} \mathrm{As} \mathrm{QW}$ 's as the thin layer of impurities varies the $z$ position. We show the maximum deformation for two well widths. The shaded area corresponds to twice the value of $\eta$. Effective atomic units (e.a.u.) are used (Rydberg constant $R^{*}=5.8 \mathrm{meV}$ and $a_{0}^{*}=100 \AA$ ).

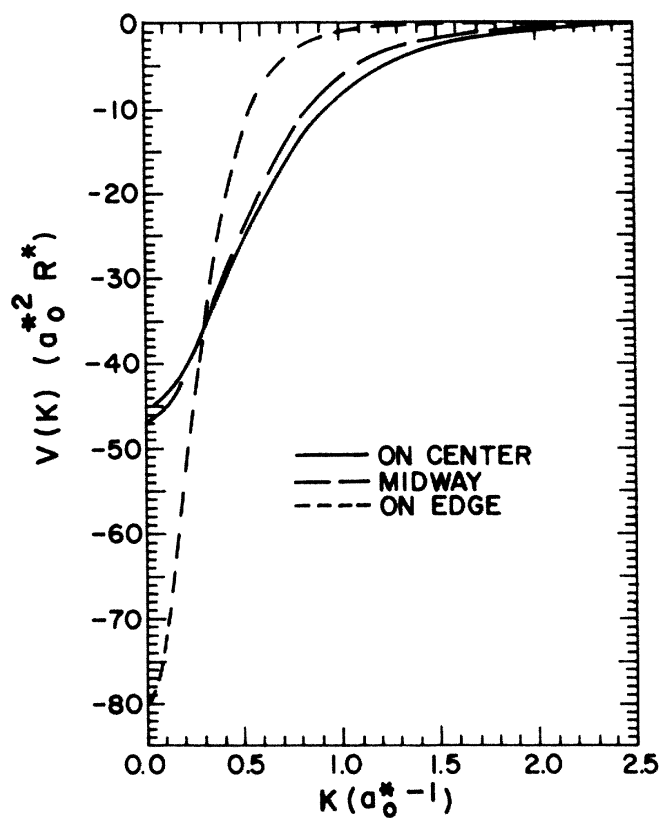

FIG. 4. Fourier transform $V(k)$ of the hopping integral calculated with the one-impurity wave functions of Bastard's model corresponding to different impurities positions inside the well ( $L=300 \AA$ ).

defined by a tolerance in $\eta$. Notice that $\eta$ measures the deformation of the impurity band, and not the effect of displacement due to different $\varepsilon_{a}$ and $\varepsilon_{b}$.

The shape of the DOS in the 2D MT theory is governed by the Fourier transform $V(\mathbf{k})$ of the transfer matrix $V_{i j}=V\left(R_{i j}\right)$. A criteria of accuracy could be defined directly on $V(\mathbf{k})$. In Fig. 4 we show $V(\mathbf{k})$ for different values of $z_{i}$. We see that $V(\mathbf{k})$ gets sharper as

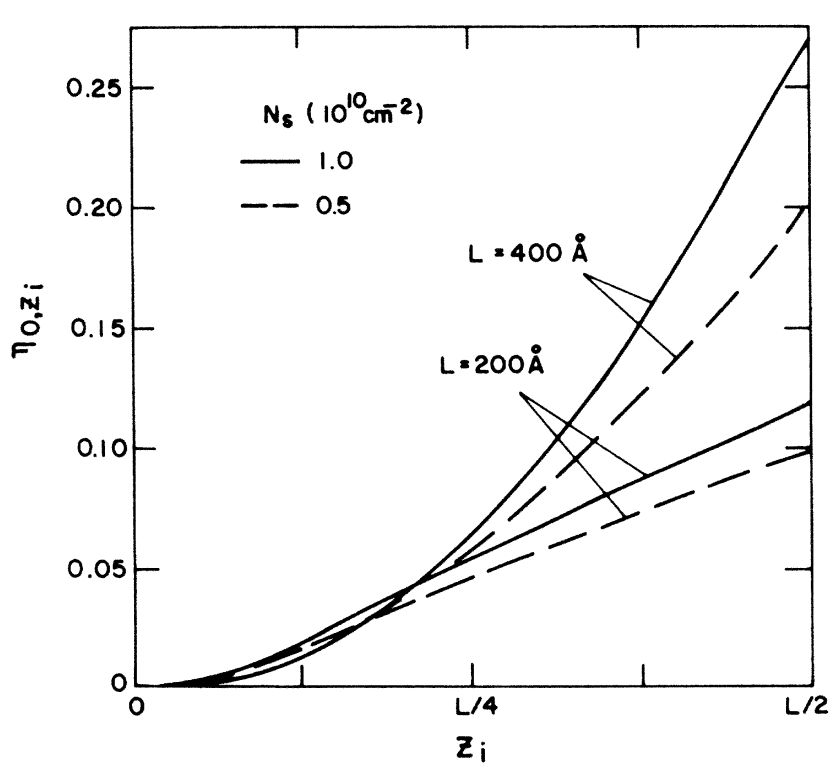

FIG. 5. Accuracy parameter $\eta_{0, z_{i}} z_{i}$. A measure of the deformation of the 2D MT DOS. 
TABLE I. Values of the relevant parameters in the range of interest for the study of the shallow states in QW's. $N_{v}$ and $N_{s}$ are given in the units of $\mathrm{cm}^{-3}$ and $\mathrm{cm}^{-2}$, respectively. $\bar{R}=\left(2 \pi N_{s}\right)^{-1 / 2}$ and $N_{v}=N_{s} \Delta$. The situations with $2 \mathrm{D}$ character are those when $1 \gg \Delta / \bar{R}$.

\begin{tabular}{|c|c|c|c|c|c|c|c|c|}
\hline \multicolumn{9}{|c|}{$\Delta(\AA)$} \\
\hline & \multicolumn{2}{|c|}{20} & \multicolumn{2}{|c|}{60} & \multicolumn{2}{|c|}{100} & \multicolumn{2}{|c|}{140} \\
\hline$N_{v}$ & $\Delta / \bar{R}$ & $N_{s}$ & $\Delta / \bar{R}$ & $N_{s}$ & $\Delta / \bar{R}$ & $N_{s}$ & $\Delta / \bar{R}$ & $N_{s}$ \\
\hline $10^{15}$ & 0.007 & $2 \times 10^{8}$ & 0.037 & $6 \times 10^{8}$ & 0.079 & $1 \times 10^{9}$ & 0.131 & $1.4 \times 10^{9}$ \\
\hline $10^{16}$ & 0.022 & $2 \times 10^{9}$ & 0.116 & $6 \times 10^{9}$ & 0.250 & $1 \times 10^{10}$ & 0.410 & $1.4 \times 10^{10}$ \\
\hline $10^{17}$ & 0.071 & $2 \times 10^{10}$ & 0.368 & $6 \times 10^{10}$ & 0.787 & $1 \times 10^{11}$ & 1.31 & $1.4 \times 10^{11}$ \\
\hline
\end{tabular}

the binding energy decreases, which corresponds to more extended states. However, since the DOS is normalizable and $\eta$ is bound to the range 0 to 1 , we prefer to use the latter criterion.

\section{RESULTS AND COMMENTS}

We present in Table $I$ the numerical values of the relevant parameters: $N_{s}$ and $N_{v}$, the superficial and volumetric impurity concentration, respectively, and $\Delta / \bar{R}$, the mentioned ratio between the impurity layer thickness and the most likely distance in the $(x, y)$ plane between two impurities. To ensure the adequacy of the 2D treatment, $\Delta / \bar{R}$ should be considerably smaller than 1. We see that for $N_{V}<10^{16} \mathrm{~cm}^{-3}$ and $\Delta<100 \AA$ this condition is quite well fulfilled. In this range $N_{s}$ goes from $10^{8}$ to $10^{10} \mathrm{~cm}^{-2}$. We will be interested in the upper part of this range, for the sake of comparison with experiments. In order to use Bastard's model we must take the well width $L$ greater than the effective Bohr radius $\sim 100$ $\AA$. Shanabrook and $\operatorname{Comas}^{6}$ worked in the range 60 $\AA \leq L \leq 450 \AA$. Another relevant parameter is the critical concentration $N_{c}$ for the metal-nonmetal transition.
For $a_{0}^{*} \simeq 100 \AA$ Mott's criterion gives $N_{c} \simeq 2 \times 10^{16}$ $\mathrm{cm}^{-3}$.

Under the above limitations, we consider an impurity layer of $\Delta=L / 2$ concentric with the well in the calculation of the averaged DOS. The condition for using Eq. (10) in that case is reduced to obtaining a value of $\eta_{0, L / 4}$ much smaller than 1 . In Fig. 5 we have plotted $\eta_{0, z_{i}}$ as a function of $z_{i}$ for two values of $L$ and $N_{s}$. Remembering that $\eta_{a b}$ describes the deformation of the 2D MT DOS as the plane goes from $z_{i}=a$ to $z_{i}=b$, we observe that the deformation in the DOS becomes larger as the well width and/or the impurity concentration increases. In the present case, the biggest $\eta_{0, z_{i}}$ corresponds to $z_{i}=L / 4$ and we see that it corresponds to a deformation of about $5 \%$. This indicates that $V_{i j}$ is not sensitive to the difference $z_{i}-z_{j}$ in the region that makes Eq. (10) a reasonable assumption.

At this point it is worthwhile to see how those 2D MT DOS's change as $z_{i}$ and $N_{s}$ vary. We have plotted in Fig. 6 two DOS sequences-one for each $N_{s}$ - of four different $z_{i}$ 's inside the well. The origin of energy corresponds to that of the electron bound to an impurity at the

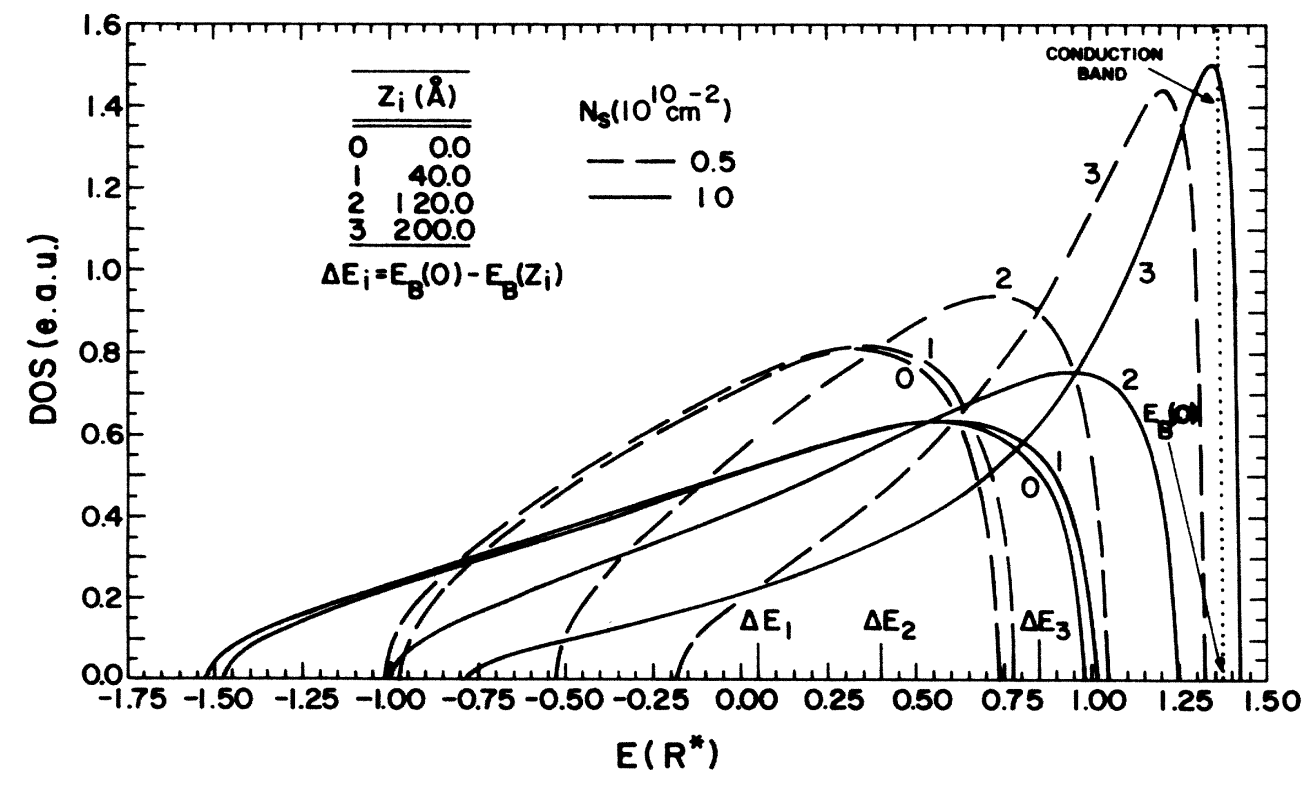

FIG. 6. 2D MT DOS as varying with $z_{i}$ and $N_{s}$ in effective atomic units. The well width is $400 \AA$ (see details in the text). 
center of the well. So, the conducting subbands begin at $E=E_{B}(0)$, the binding energy for $z_{i}=0$. As was already mentioned, $E_{B}\left(z_{i}\right)$ decreases as $z_{i}$ increases. That is why the 2D DOS for $z_{i} \neq 0$ appears dislocated by $\Delta E_{i}=E_{B}(0)-E_{B}\left(z_{i}\right)$, as assigned in Fig. 6. This dislocation is the remaining effect of the diagonal disorder on Eq. (10). We can also see that the bandwidth increases and the band gets closer to the conduction band as the impurity concentration increases. Finally, Fig. 6 gives a neat representation of the overall behavior of our accuracy parameter $\eta$ through the deformation of the 2D DOS along the well.

In Fig. 7 we compare the averaged DOS calculated in three limits: the solid line gives the pure 2D MT DOS, as those of Fig. 6, for an impurity plane at $z=0.0$, i.e., a DOS where only the off-diagonal disorder is considered; the dotted line is the $\bar{D}(\omega)$ obtained by Eq. (3) where only diagonal disorder is taken into account; finally, the dashed line is the DOS given by Eq. (10). We have assumed $P(z)=1 / \Delta$. We see that the off-diagonal disorder results in a much larger bandwidth than only the diagonal disorder. When both disorders are considered in the approximation of Eq. (10) the resulting bandwidth is the sum of the two contributions.

As the impurity concentration decreases, for a given $\Delta$, the DOS gets sharper. For $N_{s} \sim 10^{8} \mathrm{~cm}^{-2}$ the dispersion in energy is the same for both diagonal and off-diagonal disorder (the former is independent of $N_{s}$ ). The effect of the dispersion of $z_{i}$ on the DOS in Eq. (10) is basically a small dislocation of the peak $(\sim+0.58 \mathrm{meV})$ and a tail formation in the high-energy extreme. The difference in energy between the peak of the DOS in Fig. 7 and the single impurity energy is $\sim 2.6 \mathrm{meV}$. This value should be compared with the $\sim 1-\mathrm{meV}$ difference obtained experimentally; it is higher but has the same sign. This difference is very sensitive with $N_{s}$, and we are operating

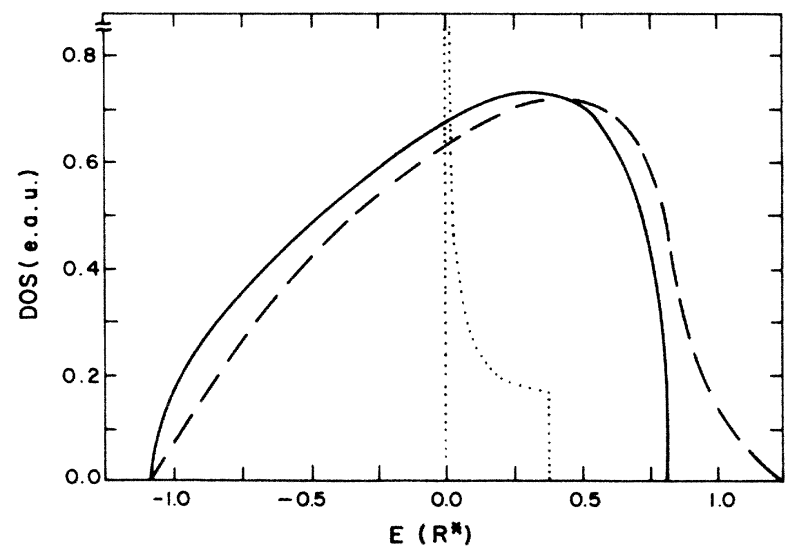

FIG. 7. Obtained density of states for the three limits: (a) considering only off-diagonal disorders, i.e., the 2D MT DOS (solid line); (b) considering only the diagonal one (dotted line); (c) taking into account both (dashed line). $L=200 \AA$ and $N_{s}=5 \times 10^{9} \mathrm{~cm}^{-2}$. Observe that in (b) the DOS is also normalized to 1 , although it has a divergence at $E=0$, represented by the open peak and the mark on the top of the DOS axis.

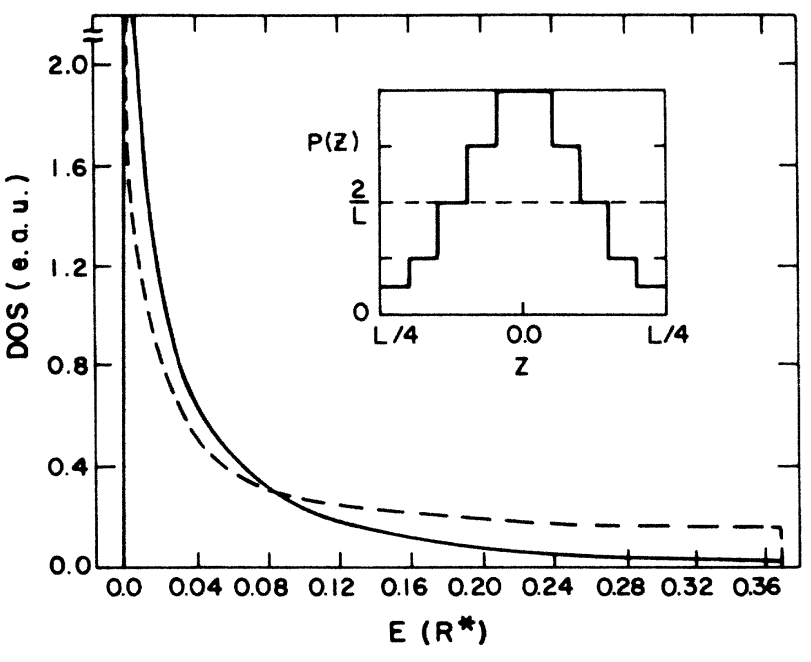

FIG. 8. The impurity profile used $P\left(z_{i}\right)$, and its effect on the DOS with only diagonal disorder as directed by Eq. (3). $L$ and $N_{s}$ as in Fig. 7.

with $N_{s}=5 \times 10^{9} \mathrm{~cm}^{-2}$, which is an upper limit of the region of interest. Besides, we are considering $P\left(z_{i}\right)$ constant.

For completeness, we have investigated the effect of introducing a nonuniform impurity profile $P\left(z_{i}\right)$. We have considered a cut-Gaussian $P\left(z_{i}\right)$ discretized as shown in Fig. 8 together with the pure diagonal disorder DOS. The Gaussian gives a sharper DOS than the uniform distribution, and slightly diminishes the contribution of the diagonal disorder as shown in Fig. 9, as expected. In consequence, the dislocation of the peak is smaller, $\sim 2.1$ $\mathrm{meV}$, getting closer to the measured discrepancy.

To conclude, we have shown that the 2D MT impurity-band treatment can also be used in a reasonable

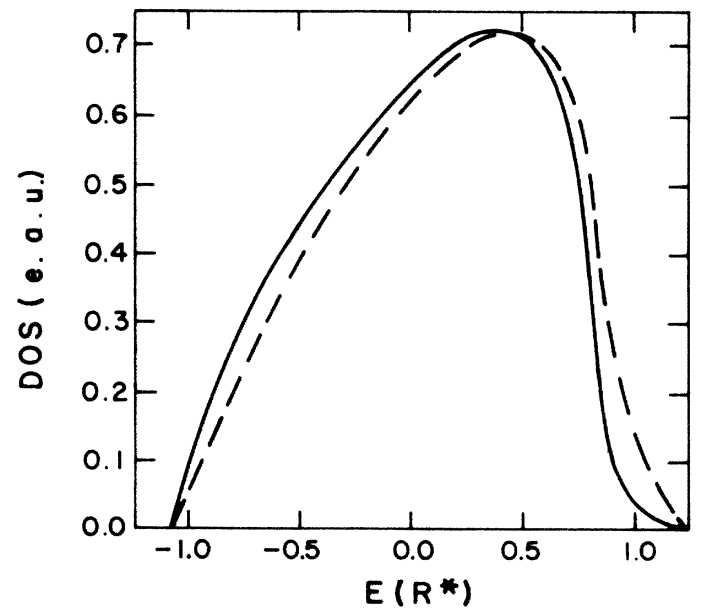

FIG. 9. The Gaussian impurity-profile effect on the $D(E)$ of Eq. (10). The dashed line gives $D(E)$ with $P\left(z_{i}\right)=$ const. $L$ and $N_{s}$ as in Fig. 7. 
way for the case of a finite-width impurity layer in QW's, e.g., $\mathrm{GaAs} / \mathrm{Ga}_{1-x} \mathrm{Al}_{x}$ As. It happens that for the concentrations and layer widths used in experiments the offdiagonal disorder plays the major role.

\section{ACKNOWLEDGMENT}

The Instituto de Pesquisas Espaciais is affiliated with the Ministério da Ciência e Technologia (MCT).
${ }^{1}$ G. Bastard, Phys. Rev. B 24, 4714 (1981).

${ }^{2}$ R. L. Greene and K. K. Bajaj, Solid State Commun. 53, 1103 (1985).

${ }^{3}$ E. A. de Andrada e Silva and I. C. da Cunha Lima, Solid State Commun. 64, 113 (1987).

${ }^{4}$ M. Tomak and V. E. Godwin, Phys. Status Solidi B 1, 137 (1986).

${ }^{5}$ L. E. Oliveira and L. M. Falicov, Phys. Rev. B 34, 8676 (1986).

${ }^{6}$ B. V. Shanabrook and J. Comas, Surf. Sci. 142, 504 (1984).

${ }^{7}$ N. C. Jarosik, B. D. McCombe, B. V. Shanabrook, J. Comas, J. Ralston, and G. Wicks, Phys. Rev. Lett. 54, 1283 (1985).

${ }^{8}$ R. Dingle, H. Stormer, A. C. Gossard, and W. Wiegmann, Appl. Phys. Lett. 33, 665 (1978).

${ }^{9}$ S. Chaudhuri, Phys. Rev. B 28, 4480 (1983).

${ }^{10}$ B. V. Shanabrook, Surf. Sci. 170, 449 (1986).
${ }^{11} \mathrm{~L}$. Ioratti and R. Tsu, in Proceedings of the Second Brazilian School on Semiconductors, São Paulo, 1985, edited by J. R. Leite and C. E. T. Gonçalves da Silva (Universidade de São Paulo, São Paulo, 1985).

12P. Lane and R. L. Green, Phys. Rev. B 33, 5871 (1986).

${ }^{13}$ T. Duffield, R. Bhat, M. Koza, M. M. Tamargo, J. P. Habison, F. de Rosa, D. M. Hwang, P. Gabbe, and S. J. Allen, Jr., Solid State Commun. 60, 557 (1986).

${ }^{14}$ E. A. de Andrada e Silva and I. C. da Cunha Lima, Phys. Rev. Lett. 58, 952 (1987).

${ }^{15}$ I. C. da Cunha Lima and A. Ferreira da Silva, Phys. Rev. B 30, 4819 (1984).

${ }^{16}$ T. Matsubara and Y. Toyozawa, Prog. Theor. Phys. 26, 739 (1961). 